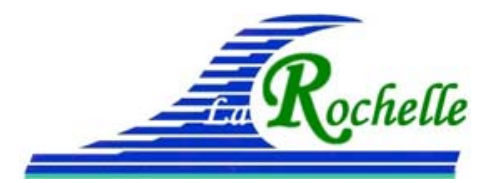

XVèmes Journées Nationales Génie Côtier - Génie Civil

La Rochelle, 29 au 31 mai 2018

DOI:10.5150/jngcgc.2018.098

(c) Editions Paralia CFL

disponible en ligne - http://www.paralia.fr - available online

\title{
Stratégies de gestion du trait de côte sur le littoral basque français
}

\section{Emilio TENA ${ }^{1}$, Caroline HUVETEAU ${ }^{2}$, Caroline SARRADE ${ }^{2}$, Nicolas PEPIN ${ }^{1}$}

1. ISL Ingénierie, 56 rue de Maréchal Harispe 64500 Saint-Jean-de-Luz, France. tena@isl.fr

2. Communauté d'Agglomération Pays Basque, 15 avenue Foch, 64185 Bayonne, France.

c.sarrade@communaute-paysbasque.fr

\section{Résumé :}

La France a acté sa stratégie nationale pour la mer et le littoral. Le document, validé par décret, est en train d'être appliqué aux différents territoires à une échelle locale (communes, agglomérations, cellules sédimentaires,...)

Le Pays Basque Français doit faire face aux risques d'érosion et submersion de son littoral. Les deux ex Agglomérations, Sud Pays Basque et Côte Basque-Adour, se sont engagées en 2012 dans l'élaboration de leur Stratégie locale de gestion de la bande côtière et des risques littoraux, déclinaison opérationnelle de la "Stratégie Nationale de Gestion Intégrée du Trait de Côte", ainsi que de la Stratégie Régionale correspondante portée par le GIP Littoral Aquitain.

Cette stratégie, fournit des éléments clés et une véritable feuille de route aux élus locaux afin de gérer le littoral de façon durable sur une échelle temporelle d'environ 25 ans.

Le rapport final de formalisation de la stratégie vise ainsi à fournir les éléments de contexte ayant conduit aux choix de gestion actés sur le territoire de la Communauté d'Agglomération Pays Basque et à décliner ces derniers sous la forme de :

- Un programme d'actions opérationnel qui récapitule les actions prioritaires à mettre en œuvre pour appliquer le scénario de gestion retenu sur chacun des secteurs de l'étude à horizon 2020 et 2040 ;

- Un programme d'actions complémentaires pour une approche globale du risque érosion.

\section{Mots-clés :}

Génie côtier, Stratégie, Trait de côte, Littoral, Erosion, Submersion, Risques côtiers, Travaux maritimes. 


\section{Thème 7 - Risques côtiers}

\section{Introduction}

La France a acté sa stratégie nationale pour la mer et le littoral. Le document, validé par le décret $n^{\circ} 2017-222$ du 22/02/2017 après plusieurs années de concertation, est en train d'être appliqué aux différents territoires à une échelle locale (communes, agglomérations, cellules sédimentaires,...). Le Pays Basque Français est particulièrement concerné puisqu'il doit faire face aux risques d'érosion et de submersion de son littoral.

Les deux ex Agglomérations, Sud Pays Basque et Côte Basque-Adour (aujourd'hui rassemblées au sein de la Communauté d'Agglomération Pays Basque), se sont ainsi engagées dès 2012 dans l'élaboration de leur Stratégie locale de gestion de la bande côtière et des risques littoraux, déclinaison opérationnelle de la stratégie régionale de gestion du trait de côte portée par le GIP Littoral Aquitain et finalisée en juin 2012.

Aujourd'hui, les $35 \mathrm{~km}$ de côtes sableuses et rocheuses constituant l'ensemble de la Côte Basque font à présent l'objet d'une unique Stratégie locale de gestion des risques littoraux partagée par les huit communes du littoral (du Nord au Sud : Anglet, Biarritz, Bidart, Guéthary, Saint-Jean-de-Luz, Ciboure, Urrugne et Hendaye) et pilotée par la Communauté d'Agglomération Pays Basque.

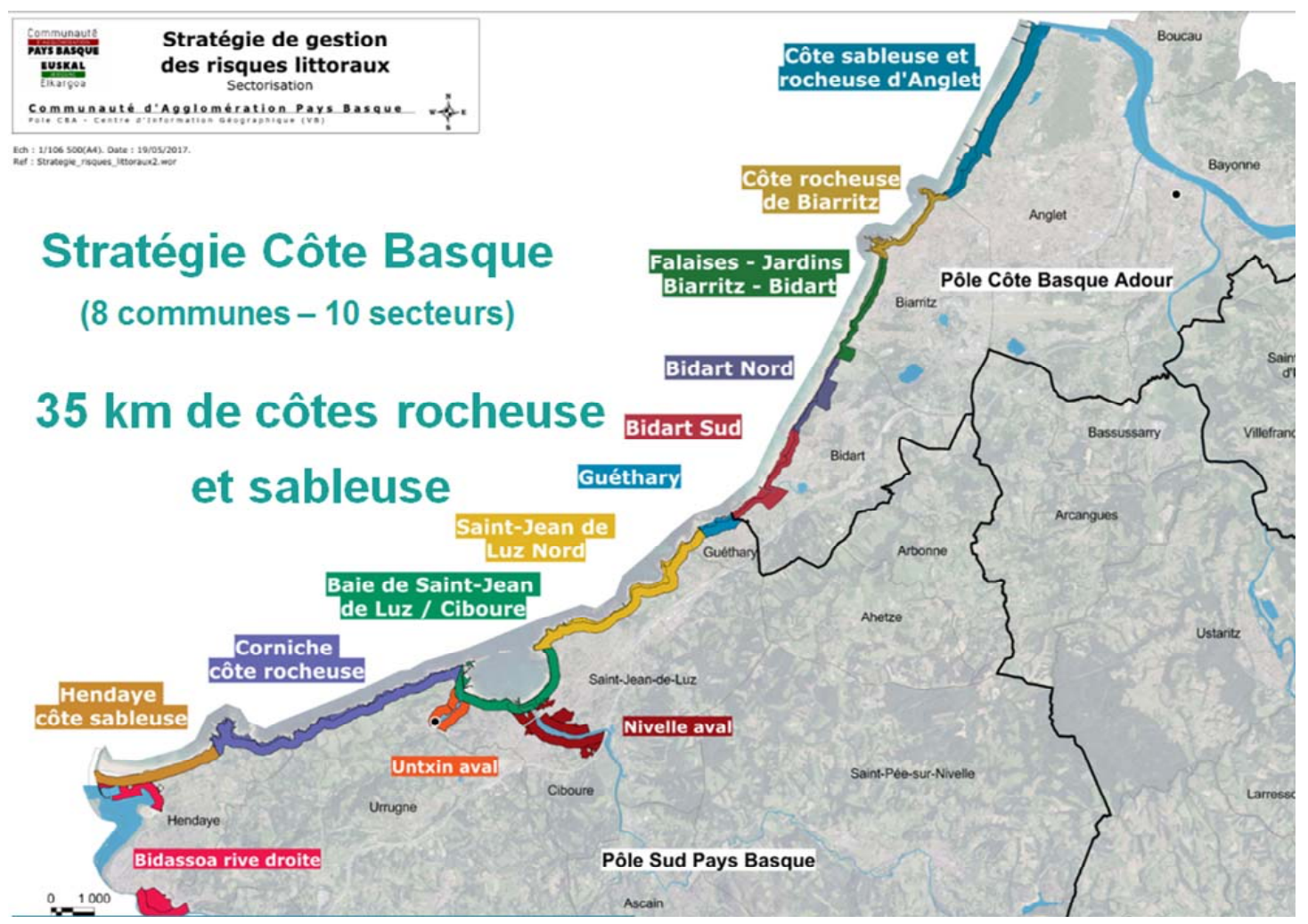

Figure 1. Sectorisation. 


\section{XVèmes Journées Nationales Génie Côtier - Génie Civil \\ La Rochelle, 29 au 31 mai 2018}

La stratégie, établie avec le concours d'ISL Ingénierie, se décline selon les grands principes de gestion suivants :

- préserver les secteurs naturels soumis à érosion,

- privilégier les actions douces sur des secteurs semi-naturels (rechargement en sable, re-végétalisation, ...) et étudier les possibilités de repli progressif de certains enjeux ponctuels (campings et golfs par exemple),

- protéger les zones urbaines les plus denses de l'érosion et/ou de la submersion pour assurer la sécurité des biens et des personnes et préserver l'activité économique (maintien des ouvrages de protection, intensification des actions de lutte active, ...),

- maîtriser l'étalement urbain dans les zones à risques et sensibiliser la population.

La gestion du littoral et des risques côtiers est une réalité complexe et multidisciplinaire qui nécessite une analyse technico-économique fine et une vision à long terme des phénomènes et problématiques (environnement, tourisme, contraintes réglementaires, contexte urbain ou naturel ou mixte, contextes géologique, hydrographique, hydrodynamique, morphodynamique, sédimentaire, activité économique, usages, etc.). Les documents produits dans le cadre de l'établissement de la stratégie locale visent donc à fournir un outil opérationnel d'aide à la décision à destination des collectivités locales (Agglomération et communes). Cette démarche, novatrice, constitue une approche de gestion concertée, responsable et durable des risques côtiers.

\section{Pourquoi une stratégie de gestion du TC?}

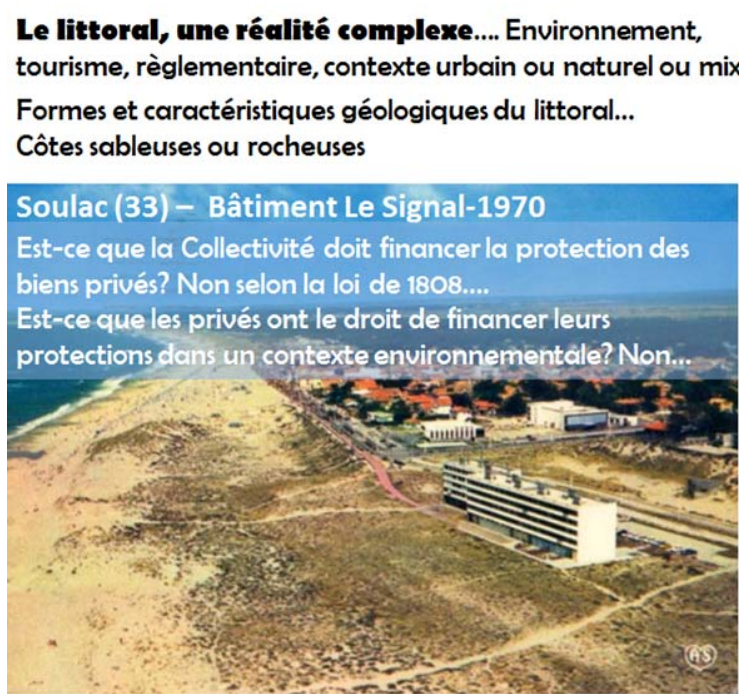

Le littoral, chiffres et enjeux 883 communes littorales et lacustres dont 785 en bord de mer ou d'océan.

- $5500 \mathrm{~km}$ de côtes métropolitaines.

- Plus de 6,1 millions de résidents dans les communes littorales métropolitaines.

- $24 \%$ du littoral métropolitain recule du fait de l'érosion côtière alors que $44 \%$ est stable.

- Nombreuses activités : nature, tourisme, plaisance, pêche..

$\rightarrow$ Tourisme littoral : $50 \%$ de l'ćconomic maritime, près de 9 milliards d'euros de valeur ajoutée et 237000 emplois.

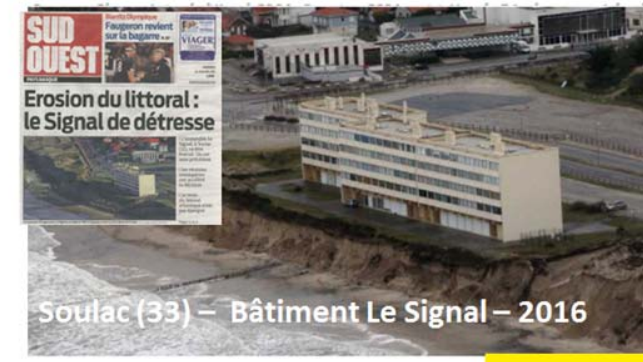

Le TC est la limite des plus hautes eaux. II se déplace et modifie les paysages.

La SGTC est un outil d' aide à la décision politique.

Elle constitue une approche Responsable et Durable.

LITTORAL AQUITAIN ${ }^{*}$ F

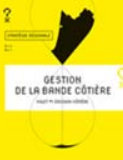

Figure 2. Pourquoi une stratégie de gestion du TC ? 


\section{Thème 7 - Risques côtiers}

\section{Données et méthodes}

Le dossier technique établi dans le cadre de la stratégie locale a fourni des éléments clés d'aide à la décision et a abouti, en 2017, à l'approbation d'une véritable feuille de route pour les élus visant à gérer le littoral basque de façon durable et raisonnée sur une échelle temporelle d'environ 25 ans.

La méthodologie, adaptée de la stratégie régionale, et développée pour élaborer la stratégie locale de gestion des risques côtiers à l'échelle de l'ensemble du territoire basque est un processus inédit en France puisque la démarche croise à la fois une expertise scientifique dans plusieurs domaines (géologie, océanologie, génie côtier, écologie, sciences sociales et économiques, géomatique, statistiques, ...) et une approche analyse coût-avantage et multicritère complète (ACA/AMC).

Le littoral basque français présente par ailleurs la spécificité de partager deux types de morphologies côtières (côte sableuse au Nord puis côte rocheuse (argiles, marnes et flyschs) sur le reste du linéaire) et d'être très exposé aux houles énergétiques (orientation défavorable et présence du gouf de Capbreton au Nord qui concentre les sollicitations).

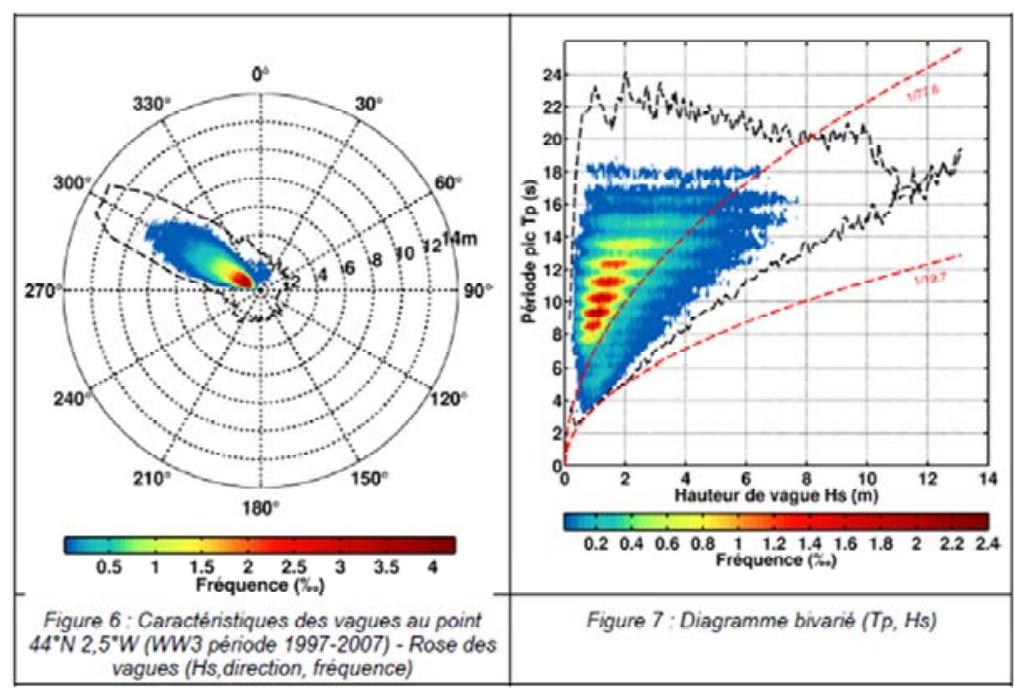

Figure 3. Caractéristiques des vagues.

Les données de base utilisées pour l'établissement de la stratégie locale ont été collectées auprès des différents acteurs locaux et regroupent :

- Documentation historique (cartes et photos anciennes);

- Données bathymétriques et topographiques (figure 1);

- Caractérisation des états de mer au large et à la côte (niveaux de la mer, houles, tempêtes,...);

- Contexte géotechnique et géologique local ;

- Contexte environnemental (cartes règlementaires notamment); 


\section{XVèmes Journées Nationales Génie Côtier - Génie Civil \\ La Rochelle, 29 au 31 mai 2018}

- Dynamique estuarienne (Adour au Nord et Bidassoa au Sud) ;

- Aléa érosion et projections du trait de côte aux horizons 2023 et 2043 (établis par le BRGM en 2013);

- Aléa submersion (cartographie des PPRL et du TRI);

- Utilisation des sols ;

- Aspects urbains (PLU, SCOT), économiques (fréquentation touristique, chiffres d'affaires, etc.) et valeur des biens (données DVF)....

\section{Résultats}

L'établissement de la stratégie locale a conduit à la production des livrables suivants :

- Un rapport de diagnostic agrémenté de nombreuses cartographies et. visant d'une manière simplifiée à répondre aux trois grandes questions suivantes :

o Comment se caractérise le littoral de l'Agglomération ?

o Comment évolue le littoral de l'Agglomération?

o Quelles sont les causes de l'érosion?

- Une synthèse des objectifs territoriaux définis par les élus politiques sur chacun des secteurs caractéristiques du linéaire d'étude et visant à orienter la définition des scénarios de gestion à prendre en compte dans la stratégie.

- Un rapport détaillant la construction et la comparaison des différents scénarios de gestion considérés sur chacun des secteurs caractéristiques de la bande d'étude (cf. figure 1):

o Le scénario "d'inaction" ou de "non gestion" est un scénario de référence défini par la stratégie régionale qui considère l'effacement complet de l'ensemble des ouvrages de protection présents sur le littoral. Ce scénario ne répond à aucune des stratégies locales fixées, dans la mesure où il est entièrement fictif, mais il permet la comparaison avec les autres scenarii dits "d'intervention". Il sert aussi à évaluer l'impact maximal sur les enjeux que l'on pourrait observer dans la zone d'étude si aucune action de gestion n'était mise en place sur le littoral.

o Le scénario "mode de gestion actuel" correspond à la continuité des actions menées par les collectivités sur le littoral jusqu'à la date d'aujourd'hui. Les modes de gestion actuels sont poursuivis sans modification et les ouvrages existants sont maintenus et entretenus sans qu'aucun nouveau confortement de grande ampleur ne soit réalisé. Pour ce scénario, ce sont 40 commerces et 529 habitas qui sont potentiellement menacés par l'érosion à l'horizon 2043.

o Le scénario "repli stratégique des enjeux" consiste à évacuer et/ou déplacer les enjeux de la bande littorale soumise à érosion. Ce mode de gestion n'intervient donc pas sur les mécanismes mêmes de l'érosion (pas de modification des taux d'évolution du trait de côte). Il autorise cependant le retour à une respiration naturelle du système littoral en redonnant de l'espace pour un fonctionnement normal. 


\section{Thème 7 - Risques côtiers}

o Le scénario "lutte active en accord avec les objectifs". A partir des objectifs territoriaux définis en phase 2 de l'étude, ce scenario de gestion vise à prioriser les secteurs où des actions de lutte active (dure ou douce) seraient à mettre en place pour assurer la pérennité des biens et des activités menacés par l'érosion, tout en restant cohérent avec les souhaits de développement des élus.

- Un rapport final de formalisation de la stratégie, dernière phase de l'étude qui vise, conformément aux indications de la stratégie régionale, à aboutir à une présentation des choix de gestion à mettre en œuvre sur le territoire de l'Agglomération Pays Basque. Cette formalisation se présente sous la forme de :

o Une synthèse des grands principes de gestion actés par les élus pour chacun des secteurs caractéristiques de la bande d'étude (choix réalisés sur la base des résultats des analyses coûts/avantages et multicritère menées en phase précédente ;

o Un programme d'actions opérationnel qui récapitule les actions prioritaires à mettre en œuvre pour appliquer le scénario de gestion retenu sur chacun des secteurs de l'étude à horizon 2023 et 2043 ;

o Un programme d'actions complémentaires pour une approche globale du risque érosion : études pour l'amélioration des connaissances, actions pour l'amélioration de la culture du risque, éléments de mise à jour des documents de prévention et des documents d'urbanisme, éléments de mise à jour des plans communaux de sauvegarde en cas de recul brutal du trait de côte pour faciliter l'alerte et la gestion de crise, etc...

Au total, le programme d'actions acté par la CAPB et qui sera décliné jusqu'en 2043, est estimé à hauteur de $150 \mathrm{M} €$ (pour les actions sous maîtrise d'ouvrage publique uniquement), soit près de 5,5 M€/an. Ce montant doit notamment être mis en perspective au regard des dommages évités par rapport au scénario de référence de l'inaction qui représentent plus de $550 \mathrm{M} €$ à l'échelle de la côte basque et à l'horizon 2043 et en ne comptabilisant ici que les enjeux monétarisables, à savoir les biens (valeur vénale des appartements et maisons), les locaux commerciaux et les voiries.

\section{Conclusions}

L'établissement d'une stratégie locale de gestion des risques côtiers à l'échelle d'un territoire aussi vaste que celui de la côte basque est un processus inédit en France. Le littoral de Communauté d'Agglomération Pays Basque est en effet partagé par des communes dont les modes de gestion historiques diffèrent nettement en fonction des caractéristiques de la commune (histoire, patrimoine, densité de l'urbanisation, objectifs de développement, activité économique, type de côte, usage des plages, morphologie des falaises, enjeux paysagers, etc.).

L'élaboration de la stratégie locale a nécessité un temps de maturation important au sein de chacune des communes et a été jalonnée par l'organisation de nombreuses réunions 


\section{XVèmes Journées Nationales Génie Côtier - Génie Civil \\ La Rochelle, 29 au 31 mai 2018}

de concertation avec les services techniques et les élus référents. Cette maturation a permis aux élus de faire progressivement évoluer leur vision du littoral et les conduit, aujourd'hui, à homogénéiser leurs pratiques de gestion à l'échelle du territoire de l'Agglomération tout en les adaptant à la réalité du risque.

La stratégie locale telle qu'elle a été établie par la Communauté d'Agglomération Pays Basque vise en premier lieu à assurer la sécurité des personnes vis-à-vis des risques littoraux. Elle a pour objectifs secondaires, le maintien de l'attractivité balnéaire du littoral (baignade, glisse, nautisme), source de la majeure partie des revenus économiques des communes littorales, tout en préservant au mieux l'environnement et la qualité paysagère des sites, tout particulièrement sur les portions de côte encore épargnés par les confortements.

\section{Littoral Pays Basque - Secteur Nord}

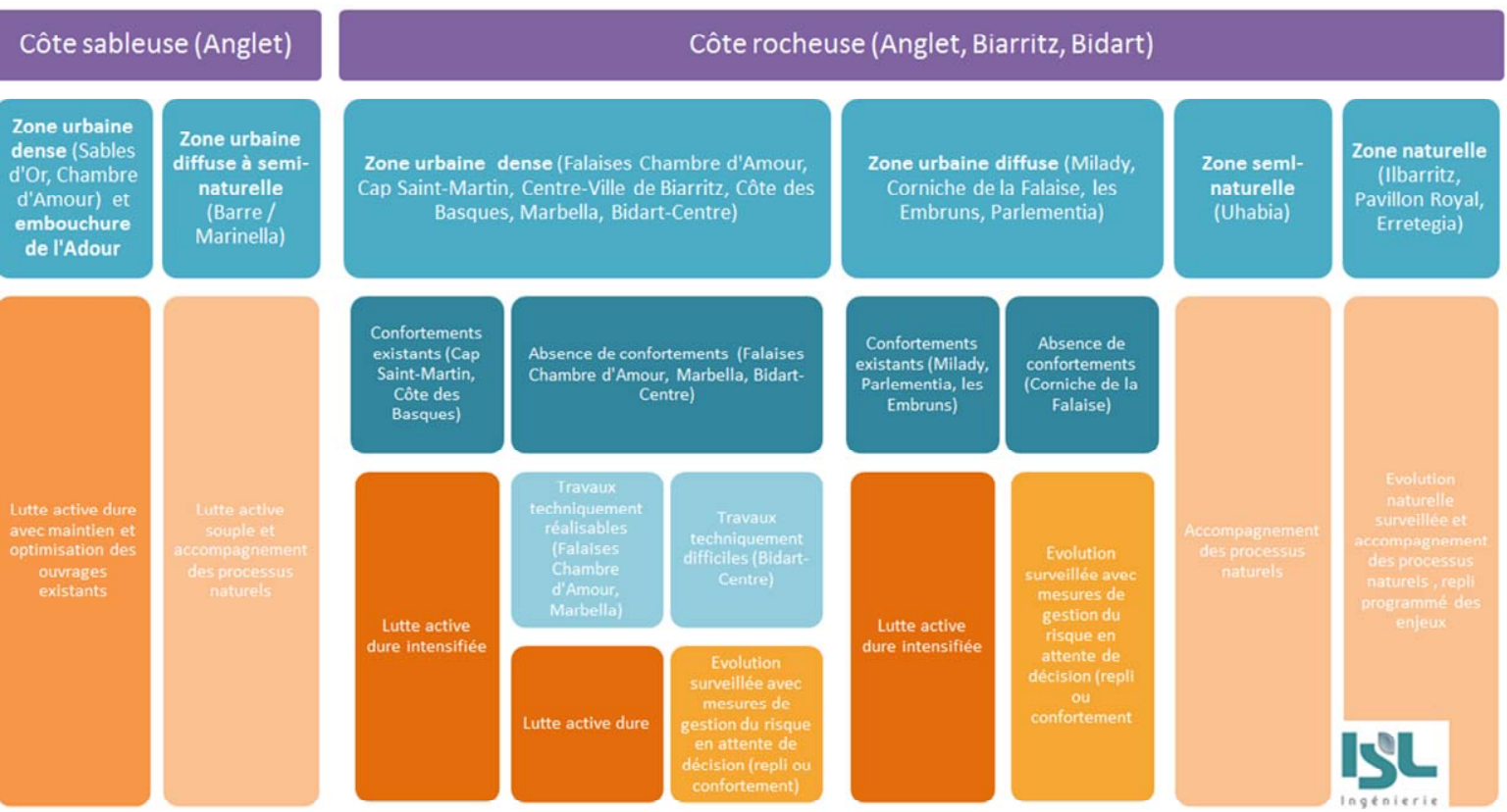

Figure 4. Synthèse du secteur Nord.

Les choix ont parfois été difficiles, notamment lorsque l'option de renforcement des falaises, nécessaire à la sécurisation des personnes, s'est opposée à la problématique environnementale et paysagère de préservation des sites ou à contrario que l'intérêt général n'était pas mis en exergue pour prévoir des actions de protection de biens privés Une cohérence a cependant été recherchée à l'échelle globale de l'Agglomération, et a permis d'aboutir aux grandes orientations décrites précédemment. Cela démontre tout l'intérêt de la méthode mise en œuvre et notamment des analyses ACA et AMC qui permettent une comparaison éclairée des différents choix de gestion qui s'offrent aux collectivités. 


\section{Thème 7 - Risques côtiers}

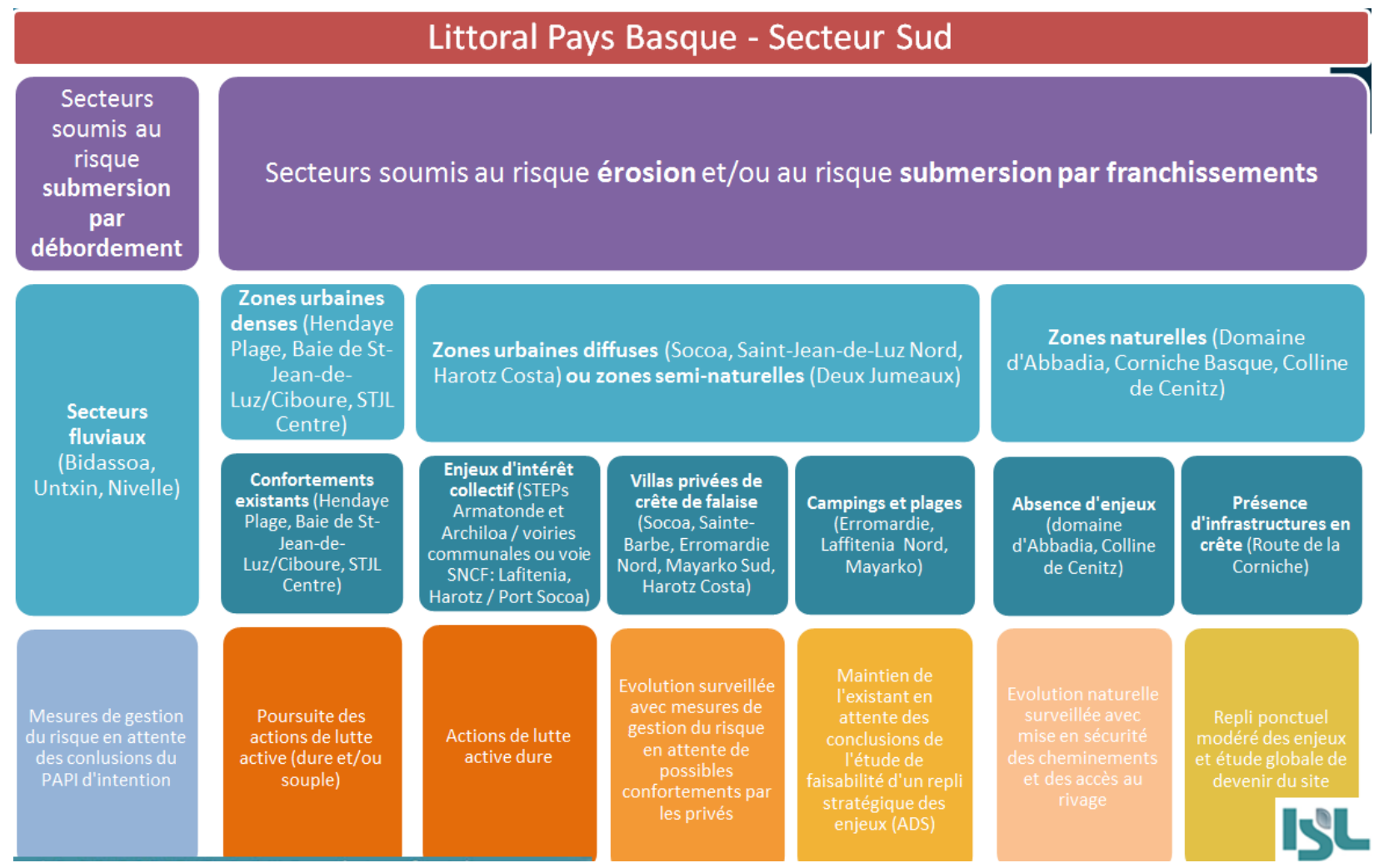

Figure 5. Synthèse du secteur Sud.

\section{Références bibliographiques}

BRGM (2013). Cartographie des surfaces inondables par submersions marines dans le cadre des Territoires à Risques d'Inondation (TRI) côtiers basques. Novembre 2013. BRGM/RP-62562-FR.

BRGM Agglomération Sud Pays Basque. (2014). Evaluation de l'aléa érosion côtière en 2023 et 2043 dans le cadre de la stratégie locale de gestion de la bande côtière. Mai 2014. BRGM/RP-63588-FR.

BRGM Agglomération Côte Basque Adour. (2014). Evaluation de l'aléa érosion côtière en 2023 et 2043 dans le cadre de la stratégie locale de gestion de la bande côtière. Octobre 2014. BRGM/RP-63780-FR.

BRGM (2015). Caractérisation de l'aléa submersion marine dans le cadre du PPRL du secteur d'Hendaye (Pyrénées Atlantiques). Avril 2015. BRGM/RP-62563-FR.

BRGM (2015). Caractérisation de l'aléa submersion marine dans le cadre du PPRL du secteur de Saint-Jean-de-Luz (Pyrénées Atlantiques). Juillet 2015. BRGM/RP-62573FR.

GIP Littoral Aquitain (2012). Stratégie régionale de la bande côtière.

MINISTERE DE L'ECOLOGIE (2010). Guide national de gestion du trait de côte.

TENA E., PEPIN N. (2015 - 2017). Stratégie de gestion du Trait de Côte ACBA ASPB. ISL - 15F003 - 15F090 - France. 